\title{
Masaoka-Koga Stage IV
}

National Cancer Institute

\section{Source}

National Cancer Institute. Masaoka-Koga Stage IV. NCI Thesaurus. Code C115034.

The tumor shows metastasis throughout the pleural and/or pericardial spaces or metastasis to distant sites. 